\title{
The Surgical Approaches of Cervical Spondylotic Myelopathy and the Predictive Factors for the Surgical Outcomes
}

\author{
Jia Liu", Xi-jing He ${ }^{1}$, Li Zhang ${ }^{2}$, Hao-peng Li ${ }^{1}$, Si-yue Xu' \\ ${ }^{1}$ Department of Orthopedics, the Second Affiliated Hospital, School of Medicine, Xi' an Jiaotong University, Xi'an, China; \\ ${ }^{2}$ Department of Digestive Disease, the Second Affiliated Hospital, School of Medicine, Xi'an Jiaotong University, Xi'an, China. \\ Email: xijing_he@tom.com
}

Received February 21 ${ }^{\text {st }}, 2011$; revised March 22 ${ }^{\text {nd }}, 2011$; accepted April $6^{\text {th }}, 2011$.

\begin{abstract}
Objective: To explore the efficacy and safety of different surgical approaches of cervical spondylotic myelopathy, and the predictive factors for the outcome of surgery. Methods: Clinical data of 68 consecutive patients who underwent surgical treatment from 2003-08-01 to 2006-12-01 were collected. The quantization of the efficacy of operation was made by applying Japanese Orthopedic Association (JOA) scoring system, based on which the recovery rate and satisfaction rate were calculated. In the patients who underwent anterior approach, we compared the recovery rate among the subgroups of different duration of symptoms, age at surgery and the severity of diseases. Any surgery-related complications were also noted. Results: $73.5 \%$ (50/68) patients underwent anterior approach, with an average recovery rate of $(68.21 \% \pm 10.06 \%)$ and the satisfaction rate of $88.00 \% ; 20.6 \%$ patients $(14 / 68)$ underwent posterior approach, with an average recovery rate of $(64.03 \% \pm 7.07 \%)$ and the satisfaction rate of $100 \%$. The recovery rate had no significant difference in the two approaches. Only 4 patients $(5.9 \%)$ underwent anterior and posterior combined approach, and the recovery rate and the satisfaction rate were $65.10 \%$ and $100 \%$, respectively. In the group of patients who accepted anterior approach, no significant differences were found in the recovery rates of different age subgroups and different duration of symptom subgroups; the significant differences recovery rates between the moderate and severe subgroups were identified. Minor complications, such as asymptomatic screw misplacement, transient dysphagia/odynophagia, pain related to the donor site and axial syndrome, were observed in a few patients. Conclusion: The JOA score can be improved by applying the appropriate approaches and the high recovery and satisfaction rates can be achieved at the same time. The efficacies of anterior and posterior approaches were similar. The complications of surgery were minor. In the patients who underwent anterior approach, the severity of diseases was a predictive factor for the outcome of surgery.
\end{abstract}

Keywords: Cervical Spondylosis, Myelopathy, Surgery, Anterior, Posterior

\section{Introduction}

Cervical spondylotic myelopathy(CSM) accounts for $10 \%$ - $15 \%$ of cervical syndrome. Its etiology is usually agerelated degenerative spondylosis. Calcification of the posterior longitudinal ligament, progressive cervical spine deformity, cervical disc herniation, trauma, spontaneous intraspinal hemorrhage, and infectious abscess are less common causes. It was reported that the ages of patients with CSM range from 20 to 90 with the median age in the mid of $50 \mathrm{~s}$. the most liable crowd are the people in their 6 or 7 decades of life, and CSM often progress during a unpredictable time course [1].

The main pathology of CSM is spinal canal compro- mise and spinal cord compression. Age-related disc degeneration leads to the collapse at the interspaces, degenerative endplate changes, osteophyte formation, arthritis, hypertrophy of the zygapophyseal and uncovertebral joints, thickening of the ligamentum flavum, and overlap and hypertrophy of the cervical facet complexes, which in turn causes the narrowing of the anterior-posterior diameter of the cervical spinal canal, the compression of spinal cord and the progressive vascular insufficiency. All these result in the cervical myelopathy and a series of symptoms. Severe symptoms, insidious onset, vulnerability of misdiagnoses and progress during an unpredictable time course are the main features of CSM. 
The treatments of CSM comprise conservative treatment and surgery. Most patients with progressive cervical spinal cord compression need a surgery which includes anterior approach, posterior approach and combined ventral and dorsal decompressive procedure. The aim of surgery is decompression of the spinal cord and elimination of the anterior-posterior flattening and distortion of the cervical cord. Realignment of the cervical spine, stabilization of cervical spinal instability, and/or correction of cervical spinal deformity can also achieved through surgical procedures. However, the choice of different surgical methods to fulfill the clinical requirements sometimes is pretty hard to make.

In this study, we explored the efficacy and safety of different approaches of surgery and the factors that affected the surgical results.

\section{Subjects and Methods}

\subsection{Subjects}

A cohort of patients were admitted to the Department of Orthopedics, the Second Affiliated Hospital of Xi'an Jiaotong University, to accept surgical treatments were enrolled in the study, whose clinical manifestations and imageology examination results were both consistent with CSM. Demography data and clinical data including main complaints and findings of physical examination were recorded.

A total of 68 consecutive patients with cervical spondylotic myelopathy who underwent surgery from 200308-01 to 2006-12-01 were reviewed, including 47 male patients, 21 female patients, with average age of 53 (range $33-71$ ), and the mean duration of symptoms was 25 months (range 1 - 120 months).

Eighty-six patients complained body and limb numbness; $73 \%$ patients suffered from lower limb hypodynamia and walking unsteadiness, among which the most severe one totally lost his ability to walk with muscle force ranked grade 2; 35\% patients complained upper limb hypodynamia and weak hand grips; 30\% patients suffered difficult defecation. In physical examination, $83 \%$ patients revealed decreased superficial sensation; 90\% patients got muscle force impairment; $86 \%$ patients showed enhanced muscular tone, $55 \%$ got attenuated superficial refluxes (including abdominal reflex and cremasteric reflex); enhanced deep reflexes (including tendon reflex, trepidation sign and ankle clonus) were found in $65 \%$ patients; Hoffmann's sign in the upper extremities and Babinski's responses in the lower extremities were found in $87 \%$ patients.

$\mathrm{X}$-ray, magnetic resonance imaging (MRI) or computer tomography (CT) of cervical vertebra was routinely performed to all the patients. The main features of image examinations were the change of curvature and alignment of cervical spine; retrogressing hyperplasia of cervical vertebra (the lip-like change of posterior and anterior edge of vertebral body), the formation of osteophytes primarily localized to the endplates and interspaces, the protrude of intervertebral disc which exceeded the posterior edge of the adjacent vertebral body, ossification of posterior longitudinal ligament, hypertrophic ligamentum flavum, decreased anteroposterior dimension of spinal canal, and the sign of spinal canal stenosis and cord compression.

\subsection{Surgical Approach}

\subsubsection{Anterior Approach}

Anterior cervical corpectomy with preserved posterior wall, autograft and plating were applied to the patients. General anesthesia with tracheal intubation or cervical plexum block anesthesia was performed. Patients are positioned supine on the operating table. A bolster was placed under the shoulders to make the heads to a modest extension. At the level of intended surgery, a curvilinear skin incision was made in a skin fold in the affected side of neck, centered over the anterior border of the sternocleidomastoid muscle. Dissection was performed down through the subcutaneous tissues and the platysma. Blunt dissection was used to dissect down to the retroesophageal space and expose the ventral aspects of the vertebral bodies and intervertebral disc. C-arm shaped X-ray was used to confirm the levels. Annular bit and nucleus gelatinosus clamp were used to remove the discs adjacent to the involved vertebra; small laminectomy rongeur was used to dissect the anterior part of the involved vertebral bodies, while the posterior vertebral wall was preserved. The posterior longitudinal ligament was also dissected. Tri-cortical autogenous iliac crest bone block was inserted in the intervertebral space after being trimmed to the appropriate shape. An internal fixation plate is then measured, contoured as necessary, and placed in the midline. Screw fixed the superior and inferior vertical bodies and the bone graft (Figure 1).

\subsubsection{Posterior Approach}

Unilateral open-door laminoplasty with suture anchor fixation was applied to each patient. General anaesthesia and tracheal intubation were performed to the patients who were positioned prone using 3 pin skull fixation on the operating tables. A midline approach is made along the nuchal ligament down to the spinous process. The paravertebral muscle is subperiosteally detached from the spinous process, and the posterior surface of the lamina is exposed precisely to the lateral mass. Osteotomy was performed on the base of the $\mathrm{C} 7$ or $\mathrm{T} 1$ spinous process, which is bent toward the hinge side to relieve tension. To 


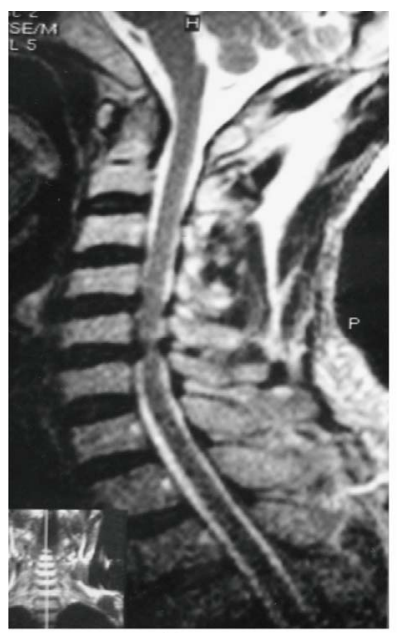

(a)

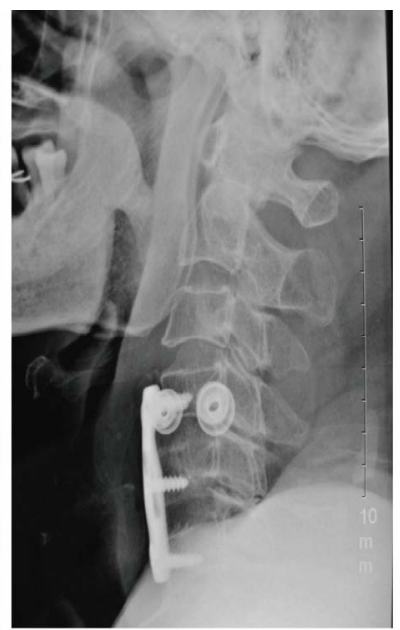

(b)
Figure 1. Anterior cervical corpectomy with preserved posterior wall, autograft and plating. (a) A 65-year old man presented with numb extremities and walking unsteadiness. Sagittal MRI scans reveal C4-C6 intervertebral disks hernia, spinal cord compression; (b) Postoperative lateral cervical radiograph reveals decompression, autograph fusion, and internal fixation at C4-C6 with restoration of alignment and lordosis.

the full-thickness of the lateral margin of the lamina of the open side and the outer cortex and of the hinge side were dissected, while the inner cortex was kept as the hinge. Connecting holes were created using a $2-\mathrm{mm}$ cutting burrin, the anchoring sutures were later passed through these holes and pull laterally. They were tied to the articular capsule to keep the opened lamina in an angle of $40^{\circ}-60^{\circ}(1.0-1.5 \mathrm{~cm}$ wide). The exposed spinal medulla was covered with gelatin sponge or fat patch incase of the postoperate accretio. In the patients with cervical spinal instability, lateral masses were inserted in the articular process as suture anchors, and bone graft was placed in the hinge side. After making sure that thorough decompression, haemostasis and secure fixation were all achieved, negative pressure drainage was placed and each anatomic layer was closed. Cervical gear was used as external fixation after bandaging (Figure 2).

\subsubsection{Combined Anterior and Posterior Approach}

Three patients underwent one-stage posterior-anterior decompression and internal fixation with bone graft procedure. The patient was under general anesthesia and prone followed by the supine position. Posterior decompression of vertebral body and fusion was carried out using titanium plank lateral mass screws. Then, the patient underwent an anterior-approach corpectomy and internal fixation with the patient's own bone graft (Figure 3). The other patient underwent two-stage anteriorposterior decompression and internal fixation with bone

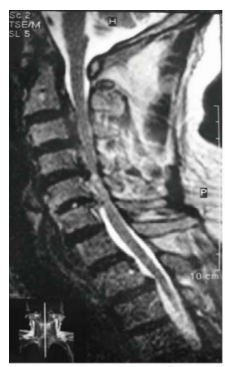

(a)

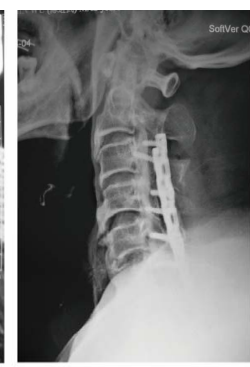

(b)

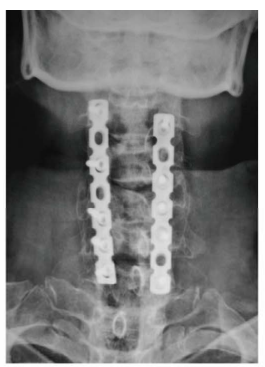

(c)
Figure 2. Unilateral open-door laminoplasty with suture anchor fixation. (a) A 63-year-old man presented with progressive quadriparesis. Sagittal MRI scans reveal C3-C6 intervertebral disks hernia and ossification of posterior longitudinal ligament; (b), (c) Postoperative cervical radiograph reveals decompression, lateral mass fixation with two 8-hole posterior plates.

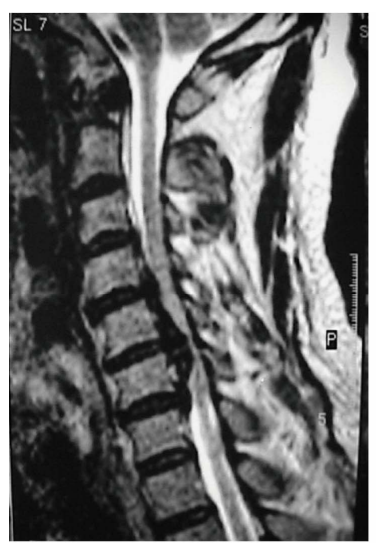

(a)

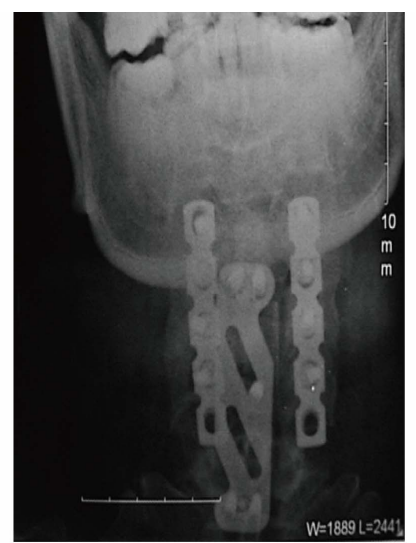

(b)
Figure 3. Combined anterior and posterior approach. (a): A 50-year-old man presented with numb extremities and progressive quadriparesis. Sagittal MRI scans reveal C3-C6 intervertebral disks hernia and spondylosis; (b): Cervical radiograph after vertebral and dorsal combined decompression reveals the autograph interbody fusion, internal fixation with anterior and posterior plates.

graft procedure. At the initial stage, anterior-approach corpectomy and internal fixation with bone graft was carried out. Three months after the anterior approach, a unilateral open-door laminoplasty with suture anchor fixation was performed.

\subsection{The Assessment of the Efficacy and Safety of Surgery}

The severity of the clinical symptoms and the outcome of treatment were evaluated using the Japanese Orthopedic Association (JOA) scoring system. A normal JOA score is 17 . The JOA score recovery rate was calculated based on the following equation: (final JOA score-preoperative JOA score $) /(17$-preoperative JOA score $) \times 100 \%$. The effect of operation was classified as very satisfied when 
the recovery rate $\geq 75 \%$; it was satisfied when the recovery rate ranged $50 \% \sim 74 \%$; it was classified as common when the recovery rate range $25 \% \sim 49 \%$; it was classified as unsatisfied when the recovery rate $<25 \%$. The satisfaction rate equals the number of patients whose recovery rate ranked very satisfied and satisfied was divided by the number of the whole patient who underwent surgical procedure. The severity of disease was classified as severe when the JOA score ranged $0-8$, which was often happened to the patient with 3 or 4 segments affected and ossification of posterior longitudinal ligament; it was classified as moderate when the JOA score ranged 9 - 12; and it was classified as mild when the JOA score ranged 13 - 16, which happened to the patient with only one or two segments affected. Any surgery-related complication was noted.

\subsection{Statistical Analysis}

JOA score was made before the surgery and 1 - 3 months post surgery, and the recovery rate and gratification rate were calculated. All the parameters were demonstrated in mean $\pm \mathrm{SD}(\mathrm{x} \pm \mathrm{s})$. Statistical analysis was performed using SPSS13.0 (SPSS Inc., Chicago, IL, USA). MannWhitney $U$ test was used for unpaired data, except for patients' gender and severities of disease were compared using one-way ANOVA analyses.

\section{Results}

\subsection{The Efficacy of Surgical Approaches}

Fifty $(73.5 \%)$ patients underwent anterior approach, 14 patients $(20.6 \%)$ underwent posterior approach. The JOA score significantly improved compared with them before the operation $(p<0.05)$. Both of the two groups got a high satisfaction rate. Between the recovery rates of the anterior approach and the recovery rates of the posterior approach, no significant difference was found. Four patients who underwent anterior-posterior combined approach, the JOA score of them improved greatly after the surgery. The sample of combined approach was too small to carry out statistic analyses (Table 1). The demographic and clinic data of patients who underwent anterior and posterior approaches were analyzed (Table 2).

\subsection{The Predictive Factors of the Outcome of Anterior Approach}

The recovery rates of patients with different duration of symptoms showed no significant difference (Table 3). No significant difference was found among the recovery rates of patients in different age subgroups (Table 4). With the aggravation of the diseases, the recovery rates and satisfaction rates declined. Significant difference was found between the severe and moderate subgroups (Table 5).

\subsection{Surgery-Related Complications}

Screw misplaced in the intervertebral space was happened to one patient who underwent anterior approach. No severe complications such as the injury of nerve root or spinal cord, or plate broke were observed. No more measures were taken except for intensive follow-up. Three patients presented dysphagia/odynophagia, which resolved 3 - 4 days later. Four patients presented pain related to the donor site, the most severe one needed analgesic to relieve the pain; and the pain lasted for 6 months for the most. Two patients underwent posterior approach, complained a worsening axial syndrome after the surgery. It presented nuchal pain, neck stiffness, and shoulder pain, and could be relieved after rest. No severe

Table 1. The relationships of different surgical approaches and effects.

\begin{tabular}{|c|c|c|c|c|c|c|c|c|c|}
\hline $\begin{array}{c}\text { Surgical } \\
\text { approaches }\end{array}$ & $n$ & $\begin{array}{c}\text { Very } \\
\text { satisfied }\end{array}$ & Satisfied & Common & Unsatisfied & $\begin{array}{c}\text { Score before } \\
\text { operation }\end{array}$ & $\begin{array}{c}\text { Score after } \\
\text { operation }\end{array}$ & $\begin{array}{c}\text { Satisfaction } \\
\text { rate }(\%)\end{array}$ & $\begin{array}{c}\text { Recovery rate } \\
(\%)\end{array}$ \\
\hline Anterior approach & 50 & 20 & 24 & 6 & 0 & $9.01 \pm 2.43$ & $14.02 \pm 1.56^{*}$ & 88.00 & $68.21 \pm 10.06^{*}$ \\
\hline $\begin{array}{l}\text { Posterior } \\
\text { approach }\end{array}$ & 14 & 2 & 12 & 0 & 0 & $7.79 \pm 2.58$ & $13.57 \pm 1.51 *$ & $100 \%$ & $64.03 \pm 7.07^{*}$ \\
\hline $\begin{array}{l}\text { Anterior-posterior } \\
\text { combination }\end{array}$ & 4 & 0 & 4 & 0 & 0 & 5.25 & 13.75 & $100 \%$ & 65.1 \\
\hline
\end{tabular}

$* p<0.05$ vs. pre-operation.

Table 2. Demographic data and endoscopic findings of the two patients groups.

\begin{tabular}{lcc}
\hline & Anterior approach $(n=50)$ & Posterior approach $(n=14)$ \\
\hline Age(years, median(range)) & $51.5 \pm 20.7(33-71)$ & $54.0 \pm 12.9(40-68)$ \\
Gender(male: female) & $34: 16$ & $10: 4$ \\
Duration of symptoms(months) & $23.7 \pm 37.6(1-120)$ & $26.2 \pm 31.8(4-84)$ \\
Pre-operation JOA score & $8.5 \pm 4.6(6-15)$ & $7.5 \pm 3.4(4-12)$ \\
\hline
\end{tabular}


Table 3. The relationships of duration of symptoms and the surgical outcomes.

\begin{tabular}{cccccccc}
\hline Duration of symptoms & $n$ & Very satisfied & Satisfied & Common & Unsatisfied & Satisfaction rate (\%) & Recovery rate (\%) \\
\hline$<6$ months & 28 & 14 & 12 & 2 & 0 & 92.86 & $68.53 \pm 7.67$ \\
$\leq 6$ months $<2$ years & 20 & 6 & 10 & 4 & 0 & 80.00 & $66.25 \pm 9.81$ \\
$\geq 2$ years & 2 & 0 & 2 & 0 & 0 & 100.00 & $67.60 \pm 3.32$ \\
\hline
\end{tabular}

Table 4. The relationships of patients' age and the surgical outcomes.

\begin{tabular}{cccccccc}
\hline Age & $n$ & Very satisfied & Satisfied & Common & Unsatisfied & Satisfaction rate $(\%)$ & Recovery rate $(\%)$ \\
\hline$<50$ years & 23 & 10 & 10 & 3 & 0 & 86.96 & $68.12 \pm 9.50$ \\
$\geq 50$ years & 27 & 10 & 14 & 3 & 0 & 88.89 & $66.94 \pm 10.35$ \\
\hline
\end{tabular}

Table 5. The relationships of the severity of disease and the surgical outcomes.

\begin{tabular}{cccccccc}
\hline Severity of disease & $n$ & Very satisfied & Satisfied & Common & Unsatisfied & Satisfaction rate (\%) & Recovery rate $(\%)$ \\
\hline Mild & 5 & 2 & 3 & 0 & 0 & 100.00 & $72.23 \pm 2.79$ \\
Moderate & 25 & 14 & 9 & 2 & 0 & 92.00 & $70.59 \pm 11.35^{*}$ \\
Severe & 20 & 4 & 12 & 4 & 0 & 80.00 & $60.25 \pm 10.36$ \\
\hline
\end{tabular}

$*_{p}<0.05$ vs. severe group.

complications such as damage of nerve root or spinal cord, leakage of cerebrospinal fluid, esophageal fistula, and damage to laryngeal nerve or recurrent nerve, neck haematoma, disunion of graft and pseudo arthritis formation were observed.

\section{Discussion}

The progress of CSM varies among different patients. In some patients, symptoms and signs progress in a gradual step-wise fashion. In other patients, symptoms and signs, once present, may stabilize without obvious progression for years, only to "reactivate" and worsen months to years later. The timing of patients with CSM undergo surgical treatment also plays a role in the management of patients with CSM. Kadanka et al. [2] ran a prospective 3 -year randomized study comparing conservative and surgical treatment of CSM. They found that patients should rather be treated conservatively if they a spinal transverse area larger than $70 \mathrm{~mm}^{2}$, were of older age, and had normal central motor conduction time, and surgery was more suitable for patients with clinically worse status and a lesser transverse area of spinal cord. While Matz [3] and his colleagues concluded through a multicenter, blinded clinical trial study, which once clinical cervical spondylotic myelopathy was evident, progression might occur despite the best of treatments, both surgical and nonsurgical. In the opinion of Jia [4] the best timing of surgery was $3-6$ months after the onset of CSM. In the study of Lu et al. [5], they found that the long-term efficacy of anterior approach surgery was closely related to the time of course, the age of onset, and suggested early anterior cervical decompression and fu- sion should be applied as long as a definite diagnosis of CSM was made. In this study, we found that age, course of disease were not a predictive factor of the surgical outcome. The severity of disease was the only factor that might leads to different outcome of surgical treatment. There was no limitation of best timing of surgery. Patients with a long history of disease can also get a satisfied surgical outcome, but they should consider the surgical treatments before they reach the severe stage of disease.

Compression of the spinal cord by the degenerating cervical spine tends to lead to progressive clinical symptoms. Surgical decompression can stop this process and lead to recovery of function. The choice of different approach depends on what is causing the compression of the spinal cord. Matz et al. [3] considered that the anterior cervical surgical approach was preferable in the treatment of CSM patients if their compressive pathology was primarily ventral, primarily localized to an interspace or interspaces, and/or was associated with cervical instability, spondylolisthesis, or a kyphotic deformity. The posterior approach should be applied to those patients with ventral pathology that extends beyond the interspace levels or with a narrow spinal canal, who cannot be treated via a dorsal only procedure because of kyphosis or instability. In their opinions it was approperate that the ventral cord compression limited to one, two, three, and rarely four interspaces. In the report of Koller et al. [6], 26 patients underwent 4- and 5-level anterior cervical fusions. They got a $100 \%$ union rate without any need for the Halo device. Their experience demonstrated that anterior-only 
instrumentations following segmental decompressions or use of the hybrid technique with discontinuous corpectomies can avoid the need for posterior supplemental surgery in 4- and 5-level surgeries. But some surgeons emphasized that Multilevel anterior decompressive procedures for cervical spondylotic myelopathy or ossification of the posterior longitudinal ligament may be associated with a high incidence of neurological morbidity, construct failure, and pseudoarthrosis. In the treatment of these patients, they chose laminectomy over anterior decompression. Some scholars held the opinion that if the cervical lordotic curvature has been well preserved, spondylostenosis or ossification of the posterior longitudinal ligament, with or without instability, may be approached posteriorly in selected older patients (over 65 years of age). In younger patients or in geriatric patients with predominantly anterior disease with kyphosis, direct anterior surgical procedures yield better results. To eliminate the disadvantages of conventional discectomy and corpectomy, cervical corpectomy with preserved posterior vertebral wall (CPW) had been performed since 1999. In the randomized control clinical study of Ying [7] and his colleagues, the efficacy and safety of CPW and conventional corpectomy were compared. Their study indicated that the improvements of JOA scores were similar in both groups, but the average operation time and blood loss decreased significantly in the CPW group. In this study, CPW was applied in all the anterior procedure, JOA scores improved significantly as compared to those before operation. In this study, we found with development of internal fixation system, 3-, 4-, even 5-level anterior fusion were the feasible procedure in the treatment of multiple segments CSM. As to the patients with hypertrophic ligamentum flavum, ossification of posterior longitudinal ligament and spinal stenosis, in our experience, decompression should be carried out posteriorly.

The posterior approach includes laminoplasty and laminectomy. Yukawa et al. [8] compared the surgical outcomes of laminoplasty and skip laminectomy, no significant differences were seen between the two groups, in terms of operative invasiveness, axial neck pain, cervical alignment, and range of motion, and clinical results in the patients of CSM without developmental stenosis. The laminoplasty includes unilateral open-door laminoplasty and bilateral open-door laminoplasty. The preference of the two different techniques is up to the experiences of the operators. Unilateral open-door laminoplasty was applied in this study, satisfied surgical outcomes were achieved. In the patients with spinal stenosis, extensive ossification of posterior longitudinal ligament or osteophyte proliferation, or segmental vertebra unsteadiness, many authors [9-11] approved that dorsal and ventral combined pro- cedure should be applied, while some reports demonstrated that simple anterior or posterior approach could also achieved satisfied decompression. Sekhon [12] treated 50 consecutive patients with circumferential spondylotic cervical stenosis with a single stage wide posterior laminectomy and lateral mass instrumented fusion, got a very low morbidity and excellent clinical and radiological outcome. In our experience, for these patients, simple anterior or posterior decompression could only relief the symptom for a short time, the application of anterior and posterior combined approach was necessary to get a thorough decompression.

To improve the fusion rate, except for autogenous bone graft, interbody cages and internal fixation plates have been used in cervical interbody fusion. There are some disputes on the efficacy of these appliances. Dai et al. [13] concluded from a randomized study that interbody fusion cage was effective in treatment of cervical spondylotic radiculopathy and/or myelopathy; the usage of anterior plate fixation could prevent cage subsidence but couldn't improve the 2-year outcome. Wright et al [14] found a high pseudarthrosis rates in anterior cervical fusions carried out at 2 or more levels without fixation, after he investigated 97 consecutive patients who accepted anterior decompressive procedures without internal fixation. They were inclined to change their practice to include internal fixation in the form of anterior plating for fusions carried out at more than one level. The study of Cheng et al. [15] also approved that the use of anterior cervical plating after anterior corpectomy and fusion with autologous bone graft greatly enhances arthrodesis. In this study, autograft and anterior plate were applied to all the patients who accepted anterior approaches, no graft misplace was observed and the fusion rate was $100 \%$. The internal plating system comprised anterior plates and posterior plates. Some study showed that multilevel laminectomy and instrumentation with lateral mass plates was associated with minimal morbidity, provides excellent decompression of the spinal cord, produced immediate stability of the cervical spine, prevents kyphotic deformity, and precluded further development of spondylosis at fused levels. In this study, 8 patients who underwent posterior decompression with unstable spinal cord, posterior mass plates were applied, satisfied surgical outcomes were achieved. We inclined that autogenous iliac bone graft was preferable to other graft substitutes, which was economic and provided a satisfied fusion rate.

There are a variety of complications related to the surgery of CSM. Screw or plate extrusion often causes adjacent organ injury. Gazzeri et al. [16] reported a delayed esophageal perforation occurred 11 years after initial radiograph revealed the screw extrusion. It migrated to the gastrointestinal tract, which caused severe dysphagia 
and high fever. The fistula spontaneous closed and the symptoms vanished at 6-month follow-up. Sahjpaul [17] reported an esophageal perforation from anterior cervical screw migration. In this case, Revision surgery was required to repair the perforation and reconstruct the cervicothoracic spine. In this study, screw misplaced in the intervertebral space happened to 1 patient. Until now, no signs of adjacent organ injury or plate extrusion were observed in the intensive follow-up. Axial symptoms occurred frequently to the patients who accepted laminoplasty. Ohnari et al. [18] found that axial symptoms were not usually so severe as to require analgesic use and did not worsen the JOA score after surgery. They believed that laminoplasty was not effective treatment for axial neck pain, and worsened it neither. In this study, 2 patient present axial symptoms, and no analgesic was prescribed. Donor site pain usually was not recognized as a significant postoperative complication. In the study of Wright et al. [14], only 2 of the 97 patients had pain related to the donor site. But in this study, it happened to 4 of 68 patients. Analgesic was prescribed to 1 patient, fortunately, it disappeared 6 months later.

On the whole, surgery was effective in the treatment of $\mathrm{CSM}$, as long as an appropriate approach was taken based on the different clinic and image presentations of different patients. Instead of the duration of symptoms, the age at surgery, the severity of disease was the main predictive factor of the outcome of anterior approach. The complications of surgery were usually scarce and minor. Skillful surgical techniques and subtle operation can avoid the incidence of severe complications.

\section{REFERENCES}

[1] P. G. Matz, P. A. Anderson, L. T. Holly, M. W. Groff, R. F. Heary, M. G. Kaiser, P. V. Mummaneni, T. C. Ryken, T. F. Choudhri, E. J. Vresilovic, D. K. Resnick and Joint Section on Disorders of the Spine and Peripheral Nerves of the American Association of Neurological Surgeons and Congress of Neurological Surgeons, "The Natural History of Cervical Spondylotic Myelopathy," Journal of Neurosurgery Spine, Vol. 11, No. 2, August 2009, pp. 104-111. doi:10.3171/2009.1.SPINE08716

[2] Z. Kadanka, M. Mares, J. Bednarík, V. Smrcka, M. Krbec, R. Chaloupka and L. Dusek "Predictive Factors for Spondylotic Cervical Myelopathy Treated Conservatively or Surgically," European Journal of Neurology, Vol. 12, No. 1, January 2005, pp. 55-63. doi:10.1111/j.1468-1331.2004.00896.x

[3] P. G. Matz, P. R. Pritchard and M. N. Hadley, "Anterior Cervical Approach for the Treatment of Cervical Myelopathy," Neurosurgery, Vol. 60, No. 1, Supp11, January 2007, pp. S64-S70.

[4] L. S. Jia, "The Status of the Anterior Approach of Cervical Spondylotic Myelopathy," The Journal of Pra- ctical Medicine, Vol. 14, No. 4, 2005, pp. 337-338.

[5] K. W. Lu, D. D. Jin, J. Wang, J. T. Chen, J. X. Wang, J. M. Jiang and D. B. Qu, "Analysis of the Long-Term Outcome of Anterior Approach Surgery on Cervical Spondylotic Myelopathy," Chinese Journal of Surgery, Vol. 44, No. 16, Aug 2006, pp.1091-1093.

[6] H. Koller, A. Hempfing, L. Ferraris, O. Maier, W. Hitzl and P. Metz-Stavenhagen, "4- and 5-Level Anterior Fusions of the Cervical Spine: Review of Literature and Clinical Results," European Spine Journal, Vol. 16, No. 12, December 2007, pp. 2055-2071. doi:10.1007/s00586-007-0398-7

[7] Z. Ying, W. Xinwei, Z. Jing, X. Shengming, L. Bitao, Z. Tao and Y. Wen, "Cervical Corpectomy with Preserved Posterior Vertebral Wall for Cervical Spondylotic Myelopathy: A Randomized Control Clinical Study," Spine (Phila Pa 1976), Vol. 32, No. 14, June 2007, pp. 1482-1487.

[8] Y. Yukawa, F. Kato, K. Ito, Y. Horie, T. Hida, Z. Ito and Y. Matsuyama, "Laminoplasty and Skip Laminectomy for Cervical Compressive Myelopathy: Range of Motion, Postoperative Neck Pain, and Surgical Outcomes in a Randomized Prospective Study," Spine (Phila Pa 1976), Vol. 32, No. 18, August 2007, pp. 1980-1985.

[9] W. B. Xu, G. Lv, L. S. Jia, D. Y. Chen, D. W. Zhao, Y. Zhu, G. J. Tu, T. Huang and J. W. Xu, "The Approach of Two Anterior Surgical Methods for Multi-Segment Cervical Spondylotic Myelopathy," Journal of Spinal Surgery, Vol. 13, No. 3, 2005, pp. 170-176.

[10] Y. S. Wang, F. Y. Zhai, L.M. Wang, G. F. Pi, L. Yin, W. D. Wang and X. J. Wu, "Selection of the Anterior or Posterior Approach Surgery for Cervical Diseases and Prevention and Cure for the Complication," The Orthopedic Journal of China, Vol. 12, No. 21-22, 2004, pp. 1611-1614. (in Chinese)

[11] C. P. Wang, M. Y. Zhai and L. Sun, "The Surgical Treatment of Cervical Spondylotic Myelopathy Using One-Stage Vertebral and Dorsal Combined Decompression, Review of 36 Cases," Journal of Zhengzhou University (Medical Sciences), Vol. 43, No. 4, 2008, pp. 830-831.

[12] L. H. Sekhon, "Posterior Cervical Decompression and Fusion for Circumferential Spondylotic Cervical Stenosis: Review of 50 Consecutive Cases," Journal of Clinical Neuroscience Vol. 13, No. 1, February 2006, pp. 23-30. doi:10.1016/j.jocn.2005.02.006

[13] L. Y. Dai and L. S. Jiang. "Anterior Cervical Fusion with Interbody Cage Containing Beta-Tricalcium Phosphate Augmented with Plate Fixation: A Prospective Randomized Study with 2-Year Follow-up," European Spine Journal, Vol. 17, No. 5, May 2008, pp. 698-705. doi:10.1007/s00586-008-0643-8

[14] I. P. Wright and S. M. Eisenstein, "Anterior Cervical Discectomy and Fusion without Instrumentation," Spine, Vol. 32, No. 7, April 2007, pp. 772-774. doi:10.1097/01.brs.0000258846.86537.ad

[15] N. S. Cheng, P. Y. Lau, L. K. Sun and N. M. Wong, 
"Fusion Rate of Anterior Cervical Plating after Corpectomy," The Journal of Orthopaedic Surgery (Hong Kong), Vol. 13, No. 3, December 2005, pp. 223-227.

[16] R. Gazzeri, M. Tamorri, A. Faiola and G. Gazzeri, "Delayed Migration of a Screw into the Gastrointestinal Tract after Anterior Cervical Spine Plating," Spine, Vol. 33, No. 8, April 2008, pp. E268-E271. doi:10.1097/BRS.0b013e31816b8831

[17] R. L. Sahjpaul, "Esophageal Perforation from Anterior
Cervical Screw Migration," Surgical Neurology, Vol. 68, No. 2, August 2007, pp. 205-209. doi:10.1016/j.surneu.2006.09.032

[18] H. Ohnari, K. Sasai, S. Akagi, H. Iida, S. Takanori and I. Kato, "Investigation of Axial Symptoms after Cervical Laminoplasty, Using Questionnaire Survey," The Spine Journal, Vol. 6, No. 3, May-June 2006, pp. 221-227. doi:10.1016/j.spinee.2005.10.014 